\title{
1 The Homeostatic Logic of Reward
}

2 Tobias Morville ${ }^{1}$, Karl Friston ${ }^{2}$, Denis Burdakov ${ }^{3,4}$, Hartwig R. Siebner ${ }^{1,5}$, Oliver J. Hulme ${ }^{1 *}$

3

$4 \quad{ }^{1}$ Danish Research Centre for Magnetic Resonance, Centre for Functional and Diagnostic

5 Imaging and Research, Copenhagen University Hospital Hvidovre, Kettegard Allé 30, 2650,

6 Hvidovre, Denmark.

$7 \quad{ }^{2}$ The Wellcome Trust Centre for Neuroimaging, University College London, 12 Queen

8 Square, London, WC1N 3BG UK.

$9 \quad{ }^{3}$ The Francis Crick Institute, Mill Hill Laboratory, London, NW7 1AA, UK.

$10{ }^{4}$ Institute of Psychiatry, Psychology and Neuroscience, Department of Developmental

11 Neurobiology, King's College London, London WC2R 2LS, UK.

$12{ }^{5}$ Department of Neurology, Copenhagen University Hospital Bispebjerg, Copenhagen, 2400,

13 Denmark

14 *corresponding author: oliverh@drcmr.dk

15

16 


\section{Abstract}

18 Energy homeostasis depends on behavior to predictively regulate metabolic states within

19 narrow bounds. Here we review three theories of homeostatic control and ask how they

20 provide insight into the circuitry underlying energy homeostasis. We offer two contributions. First, we detail how control theory and reinforcement learning are applied to homeostatic control. We show how these schemes rest on implausible assumptions; either via circular definitions, unprincipled drive functions, or by ignoring environmental volatility. We argue active inference can elude these shortcomings while retaining important features of each model. Second, we review the neural basis of energetic control. We focus on a subset of arcuate subpopulations that project directly to, and are thus in a privileged position to opponently modulate, dopaminergic cells as a function of energetic predictions over a spectrum of time horizons. We discuss how this can be interpreted under these theories, and how this can resolve paradoxes that have arisen. We propose this circuit constitutes a homeostatic-reward interface that underwrites the conjoint optimisation of physiological and behavioural homeostasis. 


\section{The problem of homeostatic control}

A remarkable feature of physiological systems is their stability. Most physiological

variables are regulated within narrow bounds by operational and computational processes

collectively known as homeostasis (Cannon 1932). The mechanistic complexity of

homeostasis extends beyond simple negative feedback control and embodies a wide

spectrum of hierarchically organised physiological control structures, molecule to agent,

operating over a multitude of timescales, milliseconds to months (Carpenter 2004).

Homeostatic control is often framed as the regulation of variables around a fixed set point,

the achievement of which upholds a physiological equilibrium (Cannon 1932). Fixed set

points, however useful they are as abstractions, are biologically implausible. Indeed,

dynamic process by which homeostatic equilibria shift. For instance moving set points could

occur through the transient modulations of stress, digestion, or arousal (Peters et al 2017),

through to longer timescales of circadian or circannual rhythms, developmental or

reproductive phases (this form of predictive regulation is also known as rheostasis and a

great many other names, see Woods \& Ramsay 2007). However, since it has been argued

that predictive control does not distinguish between allostasis and homeostasis (Woods \&

Ramsay 2007), we use the term homeostasis in this broadest sense, to encompass

predictive control. In other words, homoeostasis here subsumes classical homeostatic

reflexes and hierarchically embellished allostatic control. Under this nomenclature, the 
and overt behaviour (henceforth, behavioural homeostasis). The coordinated mechanisms of physiological homeostasis are insufficient to perpetuate survival. In an indolent (inactive or sessile) organism, the incessant activity of basal metabolic processes results in the continuous drift of vital homeostatic variables, as time passes. These excursions cannot be mitigated by the coordinated mechanisms of physiological homeostasis alone. The homeostatic error, defined as the distance of the current homeostatic state (physiological state) from any set point (Fig. 1a) can only redressed by behavioural exchange with the external environment (hunting, seeking warmth, micturition etc.). Thus, homeostatic control consists of tracking, estimating, and predicting homeostatic errors, and simultaneously prioritizing and generating the appropriate physiological and behavioural responses to minimize those errors.

In the terms defined above, this entails a conjoint optimisation of both physiological and behavioural homeostasis. From a computational perspective, this is a challenging problem for many reasons: All natural habitats are complex, uncertain, labile, and often precarious. The resources of utility for reducing homeostatic error are typically sparsely distributed in time and space. Internal states have to be inferred accurately, or at least as accurately as their survival hazards mandate. Each internal state has its own dynamics and uncertainties, so any control mechanism has to contend with variables interacting over multiple timescales, often with different degrees of synergy, antagonism, and over different scales of lag. Thus, there are rarely simple, or even stationary, mappings between the action sequences executed and the homeostatic errors minimised. 
has been approached in neuroscience. We explore how this provides insight into the logic and provenance of primary rewards with respect to homeostasis. This prefaces an empirical section, where we review the neuroanatomical basis of glycaemic and energetic control in light of recent circuit-level evidence that illustrates the predictive nature of homeostatic control circuitry, and its putative role in modulating reward value computations. We will discuss how these homeostatic networks of the midbrain and brainstem innervate dopaminergic nuclei, and modulate reward (or precision) signals, in ways that are commensurate with their homeostatic and evolutionary imperatives.

\section{Theoretical accounts of homeostatic control}

Optimal control theory. Some of the earliest models of homeostatic control emerged

91 from optimal control theory. Many of these were simple negative feedback systems where direct error correction was deployed to keep vital macro-state variables close to their setpoints (Sterling 2012; Berridge \& Robinson 2003). Common to most reactive schemes are the notions of a controller and a plant. The controller converts an input into a command which then inputs onto the plant, which outputs a motoric response, resulting in a new input. In the context of physiological regulation (Fig. 2a) the input to the controller is a homeostatic error, which is translated into a motor command. The command results in a behavioural exchange with the environment to reduce the homeostatic error. This new physiological state serves as the next input, generating the next homeostatic error. This

100 feedback control gives rise to iterative error correction. In the case of glycaemic control, the

101 homeostatic error would be inferred from the difference between current states (probed

102 through central and peripheral glucose sensors) and a euglycaemic reference state (set

103 point). The error is translated by the controller into commands for the visceromotor plant

104 and the somatic motor system. The former creates autonomic gluco-regulatory responses - 
and the latter initiates a behavioural response such as foraging and consummatory action to minimize the homeostatic error. See also Powers (2016) for treatment of control theory

107 from the perceptual side; in other words, the notion that phenomena such as homoeostasis and allostasis can be cast purely in terms of keeping sensations within bounds.

110 (Carpenter 2004). Delays or noise in the control system can lead to error hunting, which

111 results in oscillatory error corrections around the set-point. One solution is to introduce a

112 predictive component (a forward model) into the control loop. In addition to an output

113 command for the plant, direct feedback control, the controller generates an efference copy

114 that is sent in parallel to the forward model (Fig. 2b). The forward model generates a

115 prediction of the sensory state that is anticipated from the execution of the action, which

116 feeds back and is compared to the set point. This culminates in a homeostatic prediction

117 error that re-enters the controller and, if the forward model reliably predicts future

118 homeostatic error, the controller acts accordingly to minimise this anticipated error. This

119 logic can be expanded to include prediction error updates to the forward dynamic model

$120 \quad$ (Fig. 2c).

122 interoceptive signals, is the fundamental feature of predictive processing and can (in

123 principle) offer scope for explaining predictive homeostatic control outside the domain of

124 purely reactive schemes. Such predictive control structures have been deployed in

125 neuroscience to explain many phenomena, including motor control (Miall \& Wolpert 1996)

126 and awareness (Frith 2012). For comparison with the other theories we introduce below, we

127 summarise the comparator based models of control theory in the upper part of Fig. 4. 
Another feature that can be added to any of the models above is integral feedback control, where the time-integral of the error is controlled (Astrom 1995), rather than just

130 the current or projected point estimate. Integral feedback control tracks a steady-state

131 condition and only performs its regulatory action when this steady-state is violated. This

132 form of control ensures that the system variable (e.g. glucose) returns back to the set point

133 after a sustained step change irrespective of its magnitude. Integral feedback control

134 account for the control of chemotaxis in bacteria (Yi et al. 2000; Barkai \& Leibler 1997) and in systems neuroscience to explain flexibility of arousal and inhibitory control of the

136 hypothalamus (Kosse \& Burdakov 2014).

The models discussed above are deterministic in the sense that they operate under the assumption that the controller is already equipped with homeostatically rational commands

139 to issue under the spectrum of hierarchically organised errors it can receive. In other words,

140 such models do not by themselves offer any solution to the difficult problem of behavioural

141 homeostasis in an uncertain and volatile environment. Answers to questions of the sort

142 "Which sequence of actions should I perform if I want to minimise this homeostatic error?"

143 are not addressed. If one is seeking to account for the conjoint optimisation of behavioural

144 and physiological homeostasis, this is a serious limitation. Another limitation of this class of

145 model is that it provides no principled means as to how to arbitrate between commands

146 that entail different bundles of homeostatic error reductions; say between minimising one

147 unit of thermal error (e.g. $1^{\circ} \mathrm{C}$ ) and 2 units of osmolality error (e.g. $2 \mathrm{mOm} / \mathrm{kg}$ ), versus 3 and

1481 units, respectively. A seemingly sensible solution is to compute an aggregate homeostatic

149 error as the Euclidean distance from set point, and choose the action that minimises that

150 error. However, simply changing the units of measurement (e.g. from Celsius to Fahrenheit)

151 inherently imposes an arbitrary prioritisation of one homeostatic dimension over another. 
152 The aforementioned feedback control models lack any discernible principles which would

153 allow for such prioritisation to be achieved in any biologically meaningful way.

154 Drive reduction theory. The problem of coordinating and prioritising multiple

155 homeostatic feedback processes was a major inspiration to one of the earliest and most

156 ambitious attempts at modelling behavioural homeostasis; namely, drive reduction theory

157 (Hull 1943). Drive reduction theory was the first theory to algorithmically tether negative

158 feedback to homeostasis via motivational drive. Instead of direct feedback via single

159 homeostatic variables, motivational drive was proposed as a superordinate internal variable

160 that is to be minimised over the long-run.

161 Under drive reduction theory, drive compels biological agents toward actions that

162 remediate the basic physiological needs, in order to promote survival: "... when any of the

163 commodities or conditions necessary for individual or species survival are lacking, or when

164 they deviate materially from the optimum, a state of primary need is said to exist." (Hull

165 1943). Drive can thus be conceptualized as a negatively valenced state that the agent works

166 to attenuate. In so doing, the agent attenuates the associated homeostatic deficits that

167 cause it. Stimulus-response associations are reinforced as a function of the resulting drive

168 reduction - a postulate refined from Thorndike (1927). The reinforcement that accumulates

169 over time determines the strength of habitually generating a response to a given stimulus

170 (i.e., habit strength). The probability of executing a given action (i.e., the reaction potential)

171 is determined by both habit strength and drive. More complex formulations take into

172 account the inhibitory effect of fatigue, but the logic is the same. Drive-reducing actions are

173 reinforced into habits, a behavioural means by which to minimise homeostatic error. 
While drive reduction theory provides an integrated account of how deviations from

175 the homeostatic optimum motivates behaviour, the theory falls short of explaining

176 anticipatory behaviour that precedes any change in motivational drive. Animals develop

177 drive states prior to any observable homeostatic deficits such as eating when sated, drinking

178 before blood osmolality dips, and shivering before the onset of thermal challenges (Brown

179 1953; Sheffield \& Roby 1950; Seward 1956; Bolles 1968). These early experiments show that

180 the mechanistic account of drive reduction theory on learning is poorly predictive of

181 behaviour, even in narrow experimental conditions.

Homeostatic reinforcement learning. Reinforcement learning, a branch of machine learning inspired in part by behavioural psychology (and optimal control theory), offers some advance on the problem of homeostatic control. In any environment endowed with temporal regularities between sensory cues, actions, and outcomes, agents maximise expected future reward through algorithms that enable anticipatory action. The overarching

187 aim of the agent under reinforcement learning is to maximize cumulative reward over some temporal horizon (Sutton \& Barto 1998). computational role of dopamine in behavioural motivation stems from one such algorithm; discounted rewards) and the new predicted return (predicated on the outcome observed) is computed as a prediction error, which is used to update the future prediction (much like in 
prediction error hypothesis (RPE), which states that phasic firing of dopaminergic neurons in

198 the ventral tegmental area (VTA) and substantia nigra (SN) encode a reward prediction error

199 signal (Montague et al. 1996). This theoretical prediction was later experimentally

200 corroborated (Schultz et al. 1997), and since then much experimental work has underscored

201 the importance of reward prediction errors in neurobiological accounts of learning and

202 decision-making (Glimcher 2010; Niv et al. 2005). Several models have formulated

203 homeostatic control using reinforcement learning algorithms (Dranias et al. 2008; Keramati

204 \& Gutkin 2014). With respect to homeostatic control, the perspective offered by

205 Homeostatic Reinforcement Learning (HRL, Fig. 3) is interesting as it tessellates the core

206 idea of drive reduction as sketched above, with reinforcement learning (Keramati \& Gutkin

207 2014).

208 The HRL framework defines a homeostatic state space, from which a drive function is

209 derived, mapping non-linearly from homeostatic state to drive (Fig. 3, \& 4 middle). The

210 central logic is that with drive reductions defined as reward, agents that learn to maximise

211 reward, will minimise drive, which minimises homeostatic error, meaning that reward

212 maximisation and homeostatic regulation (behavioural homeostasis) are "two sides of the

213 same coin" (Keramati \& Gutkin 2014). HRL accounts for anticipatory features of behavioural

214 homeostatic control, showing that simulated agents could learn to incur short-term

215 homeostatic errors (e.g. deviations from a set point), in order to mitigate long-run (path

216 integrals of) homeostatic errors. While the HRL framework accommodates anticipatory

217 behaviour of homeostatic control, it is worth pointing out some of the residual problems.

218 Strictly speaking, HRL theory specifies no criterion to define the biological maximandum

219 (i.e., the optimal set point), but relies on experimenter-set value functions which have no

220 normative grounding. Keramati and Gutkin (2014) choose their drive function as a sensible 
and parsimonious guess based on the behavioural and economic phenomena this would entail. Interestingly they showed that several phenomena from economics and behavioural ecology could be accounted for with a simple convex drive function. To facilitate comparison between theories, HRL is juxtaposed with other models in Figure 4.

Active inference. Recent models invoke the notion of variational inference under a

Pezzulo et al. 2015). Fundamental to those formulations is the notion that the agent deploys interoception, somatic and viscero-motor actions in order to control internal states. This is principle (Friston et al. 2006; Friston 2012). Heuristically, this principle suggests that all living agents resist disorder (i.e. death) by restricting themselves to a limited number of states consistent with their physiological integrity, an idea that is consistent with homeostatic regulation as framed above, and with drive reduction theory.

Under active inference, agents stay alive by predicting the states that keep them alive, and act in order to fulfil those predictions. These predictions are generated in the higher

236 levels of the neural and autonomic hierarchies and passed down to lower levels. The lower 237 levels signal prediction errors back up the hierarchy. Prediction errors here are not about

238 reward per se, but rather discrepancies between expected and realised sensory input.

239 Sensory predictions are cascaded downwards in the hierarchy, and if it does not match

240 input, prediction errors are propagated upwards in order to update the model

241 (interoception) or act on the environment in order to change the sensory input via (motor

242 and autonomic) reflexes (Fig. 4, lower). Importantly, agents are endowed with prior beliefs

243 that are congruent with high-survival states, such as being sated, hydrated and warm. As 
such, the notion of reward - common to models of reinforcement learning and optimal said to have value.

It is useful here to compare and contrast control theoretic formulations with active inference in the proprioceptive domain, because the same principles may apply in the interoceptive domain too. In the control of striated muscle, active inference formulations of motor control replace motor commands with predictions of proprioceptive sensations.

These predictions afford the equilibrium or set points that enslave classical motor reflexes or goal-directed actions. This control architecture calls upon earlier notions such as the equilibrium point hypothesis (Feldman 1986), in which desired movements are specified in terms of equilibrium or fixed points. Clearly, as above, the question now arises: Where do the predictions or equilibria come from? In active inference, these are generated by a deep (generative) model that provides contextualised predictions that are fit for purpose, in the current context (Friston et al. 2017). In other words, hierarchically high level motor goals specify predictions of subgoals and so on - all the way down to the predicted primary sensory afferent input in the spinal cord or brain stem. The crucial aspect of this architecture is that the forward model is not used to nuance feedback control (as in comparator models of optimal control theory, e.g. Fig. 2 \& Fig. 4 upper) - it plays a foundational role in prescribing behaviour as a generative model (Fig. 4, lower). Furthermore, this architecture is effectively open loop because its set points are predefined by descending predictions. However, these predictions are generated from a hierarchical synthesis that contextualises them; rendering the overall system a closed loop architecture.

267 The argument in this paper is that exactly the same mechanisms apply in the context of 
homoeostasis through allostatic responses that rest upon purposeful behaviour in response to the interoceptive and exteroceptive cues.

271 equilibria on all levels of the hierarchy, from autonomous physiological processes to

272 behavioural homeostasis: Autonomous processes, such as the release of insulin from the pancreas when glucose levels drop, most likely constitute the lower layers in the hierarchy

274 of the homeostatic reflex arc and are most likely implemented by effector regions in the spinal cord and brainstem (Seth 2013; Stephan et al. 2016). Premeditated planning and decision-making that engenders allostatic change is governed by relatively higher layers in the control structure, e.g. in the prefrontal, insular, or anterior cingulate cortex (Stephan et al. 2016). Thus, the hierarchical structure of models suggested under active inference, has the potential to account for homeostatic regulation to unfold on all spatiotemporal scales relevant for physiological and behavioural homeostasis. It is the hierarchical architecture

281 implicit in active inference that accommodates the spectrum of spatial temporal scales; providing a hierarchal distinction between high level predictions (allostasis) and low level predictions (classical homoeostasis). In this setting, low-level interoceptive prediction errors that cannot be resolved immediately are passed to higher levels to induce deliberative behaviour that, in the long-term, returns physiology to its fixed (set) points. information theoretical (Shannon) surprise. Technically, surprise is the negative log probability of a state - which coheres with the intuition that an internal state that is highly probable - carries less surprise than one which is improbable. Importantly the level of 
occupying internal states close to set point) are most probable because they afford the

292 highest probability of survival, whereas the least probable states (the high surprise of

293 occupying extreme internal states) are the least probable because the afford the lowest

294 probabilities of survival. The high surprise states are thus the states in the tails of the

295 survival probability surface in Fig. 1b. This closely relates to another concept from

296 information theory; namely, entropy, which is simply average surprise. The overarching aim

297 of the adaptive agent is to keep sampling sensory data that is as unsurprising as possible,

298 because the agent expects to constantly find itself in homeostatic equilibria, minimising its

299 entropy. This prior belief (of being close to a set point) is engendered by a generative

300 (forward) model, yet another key concept in active inference, to which we now turn.

A generative model establishes a probabilistic map between hidden causes (internal or external states) to observed consequences (proprioceptive, exteroceptive or interoceptive sensory input) by combining a prior (here, encoding the prior probability of internal states) with a likelihood function (a probabilistic map from hidden internal states to observed sensory inputs, see Fig. 5). Principally, there are two means by which prediction error and thus surprise can be minimised. The agent can update its predictions to conform to the sensory input (interoception), or act on the world to change the sensory input generated by external states, to better match its predictions (action). The interested reader should see

310 inference in context of perception.

311 When considering homeostatic control as active inference, it is important to appreciate

312 the nature of prior beliefs. In a hierarchical setting, these are referred to as empirical priors.

313 This is because they can be informed by empirical data or sensations. This leads to a picture 
314 of the interoceptive hierarchy as encoding a cascade of prior expectations and subsequent

315 predictions for the level below. In most formulations, deeper (i.e. higher) expectations

316 usually entail longer time courses or horizons, while priors at lower levels are more

317 concerned with proximal outcomes. On this view, surprising violations (i.e., departures from

318 homeostatic set points) induce ascending prediction errors throughout the hierarchy until

319 some (allostatic) expectations change the organism's circumstances. Under this framework,

320 it is likely that some empirical priors are held with greater precision (e.g. body temperature),

321 and thus prevail with only minor modulation over many different settings, while others will

322 be lower in precision, and thus have greater latitude to be informed by context (e.g.

323 hunger). We will see later, that the precisions - afforded different prediction errors at

324 different levels of the hierarchy - are a key determinant of behaviour and the balance

325 between allostasis and classical homoeostasis.

326 In short, prior interoceptive beliefs should reflect (relatively) invariant survival

327 probabilities, and should only be (allostatically) modulated to reflect a shift in the peak

328 survival probabilities. A good example of this would be having a relatively invariant prior

329 belief about what core thermal states the agent should occupy, but then modulating this

330 under conditions of viral infection, where the survival probability function shifts such that

331 higher thermal states have the highest survival probabilities; hence the phenomena of fever,

332 and its related thermoregulatory behaviours.

Prior beliefs about homeostatic set points are likely to be hardwired in effector regions, such as the hypothalamus and brainstem nuclei. Such empirical priors are likely to be genetically specified and shaped via evolution as a function of their ability to minimise

336 surprise, given the agents respective eco-niche (Friston \& Ao 2012). On the other hand, 
priors that pertain to learning and adaption must be able to change during interaction with a dynamic, hierarchical and often volatile environment. For a more expansive account of

339 learning and homeostasis under active inference see Pezzulo et al. (2015).

340 Summary. In the above we framed the problem of homeostasis, not as a problem of

341 stability per se, but rather as predictive control over the physiological and behavioural

342 processes that keep vital homeostatic variables within the narrow (but dynamic) range that

343 ensure survival. We rehearsed some early attempts at modelling such control, using various

344 schemes of feedback control. While these may suffice for physiological homeostasis through

345 autonomous control (e.g. the baro-reflex or skeletal muscle control) they are often unstable,

346 and importantly do not afford any insight into the mechanisms of behavioural homeostasis

347 that unfold over longer timescales. Reinforcement learning solves this shortcoming by

348 proposing several algorithms that frame adaptive behaviour as reward maximisation, which

349 can be harnessed to defend a homeostatic set point (Dranias et al. 2008; Keramati \& Gutkin

350 2014). One exigent problem (see Friston \& Ao 2012 for several others) with reinforcement

351 learning in general is that the definition of reward is behaviour-centric: Agents strive to

352 maximise reward, but reward is defined from observed behaviour. Or as Berridge (2004)

353 puts it "A circular explanation is one that attempts to explain an observation in terms of

354 itself. It just reasserts what has been observed and does not really add any new

explanation." Avoiding this circularity through homeostatic considerations was a central

motivation for the development of Homeostatic Reinforcement Learning. Likewise active

357 inference accounts of adaptive behaviour avoid this circularity by providing a normative

358 account of why agents must necessarily infer and minimise surprise about their own internal

359 hidden states in order to maintain physiological integrity (Friston 2012; Friston et al. 2006).

360 This hierarchical Bayesian perspective absorbs the entire suite of concepts discussed above 
361 (see Stephan et al. 2016 for details). Concisely, set points and error functions that are

362 integral to any form of feedback control are replaced by prior beliefs (or predictions) about

363 sensory input, where subsequent deviation from those beliefs is encoded as the errors of

364 prediction (Fig. 4 lower).

Furthermore, the conceptual objects of reward and value that motivate behaviour (as

defined in reinforcement learning), are absorbed into prior beliefs about the consequences

367 of action (e.g. what actions minimise prediction errors), where desirable outcomes are simply those that engender the least surprising outcomes. So far, we have discussed active inference in general terms; in a way that places the predictions of hierarchal or deep generative models centre stage. To properly understand the implicit computational architecture that underwrites allostatic responses, it is worthwhile unpacking the imperatives for active inference in terms of resolving uncertainty. Formally, uncertainty is expected surprise. Therefore, to select policies that minimise expected surprise in the

374 future, one has to evaluate the associated uncertainty in terms of expected free energy.

375 Expected free energy usefully decomposes into epistemic and pragmatic terms - usually

376 associated with intrinsically motivated, information-seeking, epistemic behaviour on the

377 one hand and extrinsically motivated, reward-seeking, pragmatic behaviour on the other.

378 The epistemic part is important for allostatic responses (and is generally ignored in

379 reinforcement learning formulations). A simple example here is the epistemic value or

380 affordance of checking whether the fridge is contains the necessary ingredients, before

381 starting to prepare a meal, or the foraging mammal scanning its environment to infer the

382 location and habits of its prey. Typically, uncertainty reducing (expected free energy

383 minimising) policies are selected that first resolve uncertainty after which, prior preferences

384 come to dominate. This leads to a structured transition from explorative to exploitative 
behaviour. They can also be selected under satiety states, where homeostatic errors are attenuated, and the value of exploitative action is diminished. must be neuronal representations of (worldly and bodily) states in the future, under each competing policies. These counterfactual futures may have limited time horizons, but must exist under the theory. The resulting deep generative models are sometimes referred to as having counterfactual depth that necessarily entails a future. The notion of counterfactual encoding (i.e., neuronal representations of future states) is therefore something that should

394 figure, when trying to understand interoception and its role in homoeostasis (Seth 2014). given policy will resolve uncertainty and the confidence or precision placed in the ensuing beliefs about policies. In other words, to select the best policy, one has to evaluate the precision or confidence in beliefs about alternative ways forward. A body of evidence now points to dopamine as signalling fluctuations in the precision or confidence associated with

400 policy selection (Fiorillo, Tobler et al. 2003, Niv, Duff et al. 2005, Humphries, Khamassi et al. 401 2012, Friston, Schwartenbeck et al. 2014, Schwartenbeck, FitzGerald et al. 2015). This will

402 become relevant later when we interrogate the empirical evidence that speaks to different theoretical formulations of homoeostatic control. 


\section{Neural bases of energetic control}

In the following empirical section, we survey recent evidence that suggests that

407 particular circuits of the hypothalamus and brainstem play a role in predictive homeostatic homeostatic dimension is extensive and (at least relative to other homeostatic dimensions) easy to manipulate and measure. This subfield also contextualises the common use of hunger as the predominant motivational strategy for animal experiments. charged with coordinating a microcosm of homeostatic functions (Fig. 6a). The existence of opponent energy-regulating processes was an early and exciting discovery; two hypothalamic regions with opposing effects on food intake were found, a lateral area resulting in hyperphagia when stimulated ('feeding centre'), and a ventromedial area resulting in hyperphagia when ablated ('satiety centre', Aand \& Brobeck 1951; Brobeck 1946). Since then, modern cell-type specific techniques for circuit manipulation and projection-specific has afforded an unprecedented window into the deep and neuroanatomically complex networks involved in energy homeostasis. One of the major components of these networks is the arcuate nucleus (ARC), lying in the mediobasal hypothalamus, on either side of the third ventricle, just above the median eminence. There

424 also, at a finer sub-nuclei scale, opponency remains an important principle. Two cell types are found to be crucial for the control of feeding (Atasoy et al. 2012), identified by

427 (POMC), which have seemingly opposing properties. 
the nutritional state of the body, and are both necessary (Luquet et al. 2005) and sufficient

430 (Aponte et al. 2011) to evoke voracious feeding and food-seeking behaviours: the number of

431 stimulated AgRP neurons is linearly predictive of food intake. These effects appear to be

432 mediated by GABA and the neuropeptides NPY and AgRP, that stimulate food intake when

433 delivered directly to the arcuate nucleus. In the absence of food, stimulation of AgRP

434 neurons promote a range of learned behaviours that relate to hunger and food-seeking

435 (Dietrich et al. 2015). POMC neurons by contrast are activated by energy surfeit and their

436 activity inhibits food intake and promotes weight loss (Atasoy et al. 2012). AgRP and POMC

437 neurons are both regulated by the circulating endocrine signals of nutritional state,

438 modulating their activity in mutually opposing directions consistent with their function.

439 These two cell types interact in part through a common set of downstream melanocortin

440 expressing neurons that are activated by POMC and inhibited by AgRP. These two

441 subpopulations are interspersed within the ARC making it an obvious candidate site for the

442 encoding prediction errors for energetic wealth (energy balance, or other synonyms).

443 Predictive responding. A recent stream of research has employed cell-specific

444 techniques to image and causally manipulate the activity of AgRP neurons under different

445 homeostatic challenges that each manipulate homeostatic error, and thus causally control

446 the motivational state of the animal. Natural deprivation, ghrelin injection, pharmacological

447 or optogenetic activation of AgRP neurons evoke voracious feeding and inhibit POMC

448 neurons, as might be expected with a large deviation from a set point (Betley et al. 2015;

449 Chen et al. 2016; Krashes et al. 2014; Mandelblat-Cerf et al. 2015). However, a homeostatic-

450 comparator based view of the hypothalamus has been challenged by several recent papers 
that show AgRP and POMC neurons encode predictive signals, varying as a function of seeing the food? Emphasis on the surprising nature of this result, now replicated several times, hinged on the fact that inhibition occurs even before the food is tasted. Yet, we would argue that the predictive nature of the signal, does not rest on it occurring before the taste of the food, since even if it were time-locked to the taste at consumption, it would still be predictive insofar as no change in nutritive wealth is yet manifest. Indeed, any candidate drive or hunger signal that changes reliably to extero- or interoceptive cues is still a predictive signal with respect to the slow dynamics of the gastro-intestinal cascade (the

468 (like taste or olfaction), as they are to the exteroceptive signals underpinning cue-learning

469 (e.g. sight). Since these responses are not taken to be paradoxical, it could be said that the sensory paradox somewhat dissolves. 
quality, caloric content (Chen et al. 2016), and even show a rebound back to original levels as a function of the agents beliefs, are mirrored by the hormonal signals of energetic status, that can also be considered endocrine predictors of future energetic wealth: Leptin, putatively signalling positive energetic wealth (Domingos et al. 2011), suppresses AgRP signalling its converse, excites. The inhibition appears sensitive to the appetitive affordance of food (Gibson 2001), such that food presentation in a closed container that allowed sight and smell of food but not consumption, had diminished inhibitory effects (Chen et al. 2016). 
gastrointestinal and adipose systems. The way that energetic wealth predictions are derived from these redundant signals will be an important next step toward understanding the AgRP encoding function.

It is interesting to note that the AgRP responses are heterogeneous in their temporal

502 kinetics (Betley et al. 2015), in responding to food-predictive cues, with some showing a slow attenuation over time, and others faster. This suggests that the AgRP population as a whole encodes a distribution of energetic errors over a spectrum of temporal horizons. and drive reduction theory as outlined above, is that both drive and AgRP carry negative

507 valence, as well as the fact that reducing-drive and reducing-AgRP activity are imbued with

508 positive valence. This is a subtle issue, and can cause some seemingly conflicting

509 conclusions, with some groups reporting that AgRP carries negative valence (Betley et al.

510 2015), and others reporting its positive valence (Chen et al. 2016). The discrepancy can

511 arguably be resolved in light of DR. Under DR (and therefore its cognate, HRL), drive is a

512 negative valence signal, that agents work to minimise. Actions that reduce drive are

513 rewarding which reveals why the attribution of valence to neural signals could easily be

514 conflated. The key prediction is that if AgRP signals future drive (an error on the predicted

515 energetic wealth), then AgRP stimulation, in the absence of any means of reducing drive (i.e.

516 food), should be aversive since drive-inflations are costs (negative reward). Indeed, AgRP

517 stimulation can condition place (and flavour) aversion (Betley et al. 2015). However in the

518 presence of food, the drive reduction that follows AgRP stimulation should be larger and

519 thus more rewarding, thus the reinforcing effect of AgRP stimulation should only occur in the presence of food, which is indeed what is observed (Chen et al. 2016). This is indeed a 
521 key distinction between the two opposing papers. One apparent problem with this model, is

522 that mice fail to perform operant responses in order to shut off AgRP neuron activity (Betley

523 et al. 2015; Chen et al. 2016); however, it is important to consider issues of credit

524 assignment. Under natural conditions, a drive reduction such as that associated with AgRP

525 silencing, in the absence of sensory food cues, can only be due to post-ingestive effects. This

526 means that the food consumed minutes or hours previously will be assigned the credit for

527 the drive-reduction caused by AgRP inhibition now, which predicts that recently performed

528 operant actions should not necessarily be reinforced at all. Under the mouse's generative

529 model of the world, (again in the absence of sensory food cues) the drive reduction should

530 most likely be caused by actions/sensory/gustatory events long before the operant action

531 was performed. How easily mice could learn this long-range temporal contingency with

532 overtraining though is an open question.

533 Interface between reward prediction errors and glycaemic control

$534 \quad$ Introducing dopamine. The catecholamine dopamine is synonymous with

535 reinforcement, reward and motivation. Whilst the literature on dopamine is vast, we will

536 restrict discussion to its putative role in glycaemic or energetic control as discussed above. It

537 is well known that phasic signals in ventral tegmental area (VTA-DA), and thus dopamine

538 release in the mesolimbic system, systematically scale with the nutritive value of oro-

539 sensory events in monkeys, where reward magnitude is determined by the volume of

540 nutrients consumed (Tobler et al. 2005; Stauffer et al. 2014; Ballard \& Knutson 2009). In

541 humans, there is evidence that post-prandial dopamine release is modulated by deprivation

542 with dopamine binding decreasing more in response to consumption after fasting compared

543 to non-fasting (Small et al. 2003). This echoes extant evidence from rats and mice that show

544 increased dopaminergic release (as measured by dopamine metabolite 3,4- 
dihydroxyphenylacetic acid) at feeding after a period of starvation in the nucleus accumbens

546 (NAc, McCullough \& Salamone 1992; Radhakishun et al. 1988), medial prefrontal cortex (but

547 not NAc, Carlson et al. 1987) and interestingly, the posterior hypothalamus (Heffner et al.

548 1980). Despite these findings and others, one of the curious features of the literature on

549 phasic DA and reward is that animals are motivated by a homeostatic deficit such as thirst or

550 hunger, and yet homeostatic states are rarely foregrounded in analyses of relevant

551 modulators of reward signalling. One recent interesting exception to this is offered by Cone

552 and colleagues, who present evidence for how sodium depletion can modulate RPE in the

553 NAc of rats (J. J. Cone et al. 2016). By pairing sodium sated and depleted rats with

554 conditioned and unconditioned stimuli, they found that phasic dopaminergic RPE signals can

555 manifest independently of learning and are "expressed as a function of their current

556 [homeostatic] value to the organism" (J. J. Cone et al. 2016, square brackets added). signals. As the animal plays its task for consumption of water or sugar-containing juice, its homeostatic deficits diminish, or are predicted to diminish, meaning that the value of those commodities should steadily decrease. Indeed, given the quantitative evidence for a relation between RPE and marginal utility (Stauffer et al. 2014), the fact that this is rarely tested or acknowledged (or for that matter controlled for) is surprising, given that the manipulation that makes the outcomes rewarding is continually being attenuated, until the animal rejects

564 further play, presumably because the marginal utility of consumption has depleted to a point of indifference. For this reason, we recommend greater scrutiny of homeostatic

566 states, and their dynamics under neurobiological studies of reward. In the case of energetic

567 variables, intra-arterial telemetric glucose monitors are now available, and could afford

568 important insights in this regard. 
At this point, we introduce a fundamentally different perspective on the role of

570 dopamine. In schemes that commit themselves to some form of reinforcement learning,

571 dopamine is usually cast as a reward prediction error (Fig. 4, middle); namely, the difference

572 between expected and encountered reward. This is in contrast to active inference

573 formulations, which accommodates the fact that dopamine is a neuromodulator. In other

574 words, dopamine cannot drive synaptic responses - it can only modulate them. This

575 modulatory role is exactly that required of precision. On this view, phasic dopamine responses signal an increase in the precision or confidence placed in beliefs about ongoing

577 policies. For example, the transfer of dopamine responses from unconditioned to conditioned stimuli reflect the increase in confidence about "what I should do" after observing a conditioned stimulus. In short, the reinforcement learning (reward learning) story associates dopaminergic responses with RPE, while the active inference story treats dopaminergic function as encoding the confidence in policy selection, based upon inferred states of the world. interface between homeostatic variables and reward or precision computations, there is convergent (but still tentative) evidence to suggest how the interface could be implemented (Fig. 6a \& 6b). VTA-DA neurons host a number of receptors that would mediate this interface; they are positively modulated by ghrelin, a hormone reporting short-term energy deficits, and melanocyte-stimulating hormones $(\alpha, \beta, \gamma)$ released from POMC neurons; whereas they are inhibited by AgRP and its co-transmitter GABA, as well as by hormone

590 insulin, and leptin, as well as by GLP-1 (Ferrario et al. 2016). Thus, the cells themselves

591 provide ample opportunity for interfacing from homeostatic state information to the 592 precision or reward value signal that is broadcast to the mesolimbic system from the VTA. 
594 directly to both the VTA and the substantia nigra (Dietrich et al. 2012), and POMC neurons

595 have been labelled by retrograde tracers in the VTA (King \& Hentges 2011, Fig. 7). On the

596 reinforcement learning account AgRP, neurons should positively modulate VTA-DA, since

597 they encode something hunger-like and hunger increases the value of food. Conversely,

598 POMC neurons, encoding the converse of AgRP, should then negatively modulate VTA-DA.

599 In fact, the opposite appears to be observed. As noted above, AgRP neurons exert

600 inhibitory effects over VTA-DA cells, directly via inverse agonism of the MCR3 receptor (the

601 predominant melanocortin receptor expressed on VTA-DA neurons), and indirectly via its

602 co-transmitter GABA, that acts to stimulate inhibitory interneurons that inhibit VTA-DA cells.

603 Symmetrically, POMC neurons release melanocyte-stimulating hormones which also

604 activate MCR3, which activates the VTA-DA neurons. These empirical results fit comfortably

605 with active inference in the following sense: If AgRP neurons encode the hypothesis that "I

606 need to eat", then higher level (allostatic) expectations about eating will suppress their

607 activity. However, the higher-level expectations that "I am about to eat" must be held with

608 confidence or precision that is accompanied by dopaminergic discharges. In short, when

609 AgRP firing is suppressed this will necessarily entail a confidence belief that "I am about to

610 eat" and a disinhibition of dopaminergic outflow to the cortical basal ganglia thalamic

611 systems responsible for policy selection.

612 Why the counterintuitive responding? Taken in the context of the predictive control

613 findings discussed above if AgRP is deactivated, this means that under our interpretation,

614 the precision on the prediction of positive future energy wealth increases, which is encoded

615 via phasic dopamine, via the release of VTA-DA from inhibition. Likewise, if the POMC 
616 neurons are simultaneously activated by the same sensory evidence, then this has an

617 excitatory effect on the same VTA-DA cells, which together with the AgRP disinhibition,

618 provides a means by which VTA-DA signalling can be anchored to updates of predictions on

619 future energetic wealth (i.e., the consequences of beliefs about the current long-term policy

620 are assigned high precision or confidence). This is corroborated by ex-vivo recordings in

621 which VTA-DA neurons increase baseline firing to injections of $\mathrm{Y}$-MSH (Pandit et al. 2015). It

622 should be noted that these findings seem to be at odds with the existing consensus that

623 AgRP neurons acts to increase feeding and reward, and MSH acts to decrease feeding (Yen

624 \& Roseberry 2015).

This might however be an artefact of the way these injection experiments are performed. Pandit and colleagues (2015) show that infusion of a non-specific MCR agonist that targets both MC3R and MC4R, then sucrose responding decreases (also shown by Shanmugarajah et al. 2017; Yen \& Roseberry 2015). However, by adding an MC4R antagonist, turns this response into an increase in sucrose responding. The important point

630 here being that MC3R is predominantly expressed in the VTA, whereas the MC4R is

631 expressed more broadly (for instance in the Nucleus accumbens) but not in the VTA.

632 Interestingly the MC3R are predominantly expressed on the D2R expressing neurons that

633 project into the nucleus accumbens. Notably the increased sucrose responding mediated by

634 MC3R receptor agonism, is dependent on dopamine since DA-antagonism eliminates the

635 effect (Pandit et al. 2015). Together, this might explain the apparent contradictions between 636 prior work showing that melanocortin injections decrease responses to food reward (Yen \& 637 Roseberry 2015). 


\section{Conclusion}

640 In addressing the problems of homeostatic control, we have tried to bridge between

641 several different fields, from evolutionary theory, neo-behaviourism, reinforcement

642 learning, and computational and metabolic neurosciences. In doing so, this paper offers two

643 main contributions.

644 First, we revisited how control theory and reinforcement learning have been applied to

645 motivational behaviour, reward and homeostasis. We marshalled existing as well as novel

646 arguments, for how these schemes rest on biologically implausible assumptions, either via

647 circular definitions of reward, unprincipled groundings of value or drive functions, or by

648 assuming degrees of certainty that are incompatible with the capricious nature of our

649 natural habitats. Against this background, we have reviewed the active inference framework

650 as it applies to these same homeostatic control problems. Putatively, we conclude that this

651 offers promise in circumventing the shortcomings summarised above, and at the same time

652 retains and builds on several important notions from comparator-based and reinforcement

653 learning models. Of these conceptual advances, the most important are that set points are

654 absorbed into prior beliefs about hidden viscero-sensory states, that homeostatic errors are

655 cast as precision-weighted errors on interoceptive predictions, and that optimal choice

656 behaviour is framed as an inferential process given a generative model of the body and its

657 environment.

658 Second, we reviewed extant evidence pertaining to the how homeostasis interfaces

659 with value computations in the domain of nutrient energy. Focusing on the case of the

660 arcuate nucleus, we reviewed recent evidence for its role in the predictive control of energy

661 homeostasis, contextualising the observations in the context of competing theoretical 
662 formulations. We assembled evidence suggesting that a subset of these arcuate

663 subpopulations project directly to, and are thus in a privileged position to opponently

664 modulate, dopaminergic VTA cells as a function of energetic predictions over a spectrum of

665 temporal horizons. Further, we have surveyed how circulating factors that contribute to the

666 dynamics of glucose homeostasis are direct modulators of dopaminergic neurons in the

667 midbrain as well. The emerging picture points to a multi-faceted homeostatic-reward

668 interface between the hypothalamus and midbrain. This interface may play a pivotal role in

669 the conjoint optimisation of physiological and behavioural homeostasis.

670 That said - given the current state of knowledge - assigning computational roles to

671 hypothalamic neurons may be premature. The computational quantities entailed by active

672 inference are many, and their differences can be subtle. For instance, whether AgRP or

673 POMC are encoding predictions, prediction errors, interoceptive states, or precisions

674 portended by those states, will require careful experimentation. Existing evidence does not

675 yet conclusively support one or the other. However, we hope that the theoretical

676 perspective offered here motivates empirical experiments that can disambiguate between

677 computational formulations of the brain's homeostatic-reinforcement interface. 
a.

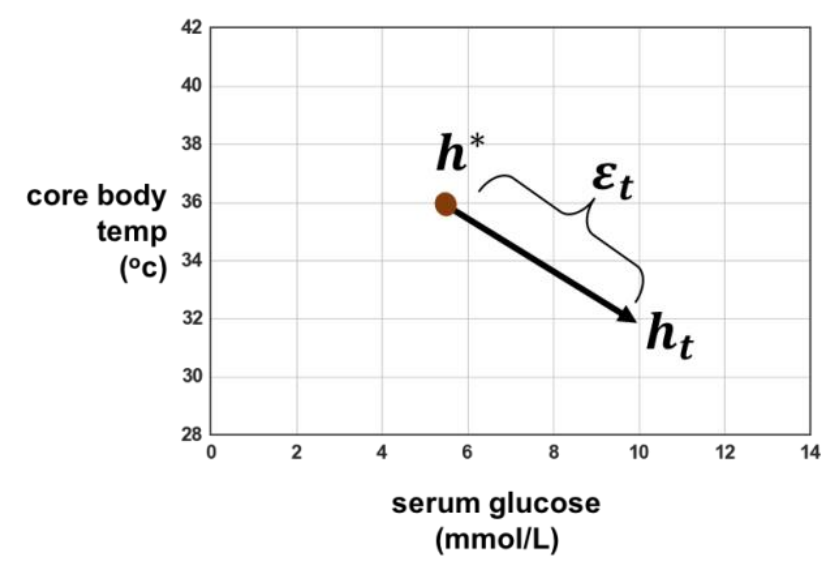

b.

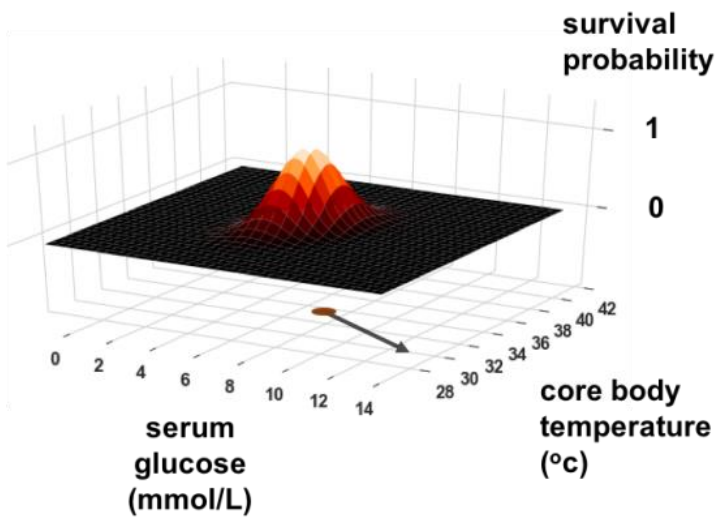

680 Figure 1| Homeostatic state space and survival probability. a, Schematic showing a simple 2-dimensional

681 homeostatic state space, where $h^{*}$ denotes a set point, $h_{t}$ current state at time $t$, and the error between them

682 defined here as the absolute Euclidean distance $\varepsilon_{t}, \mathbf{b}$, Shows a survival probability surface, depicted over the

683 same homeostatic state space, thus highlighting the relation between homeostatic error and the conditional

684 probability of survival (over some time interval), given the occupation of that homeostatic state.

685 


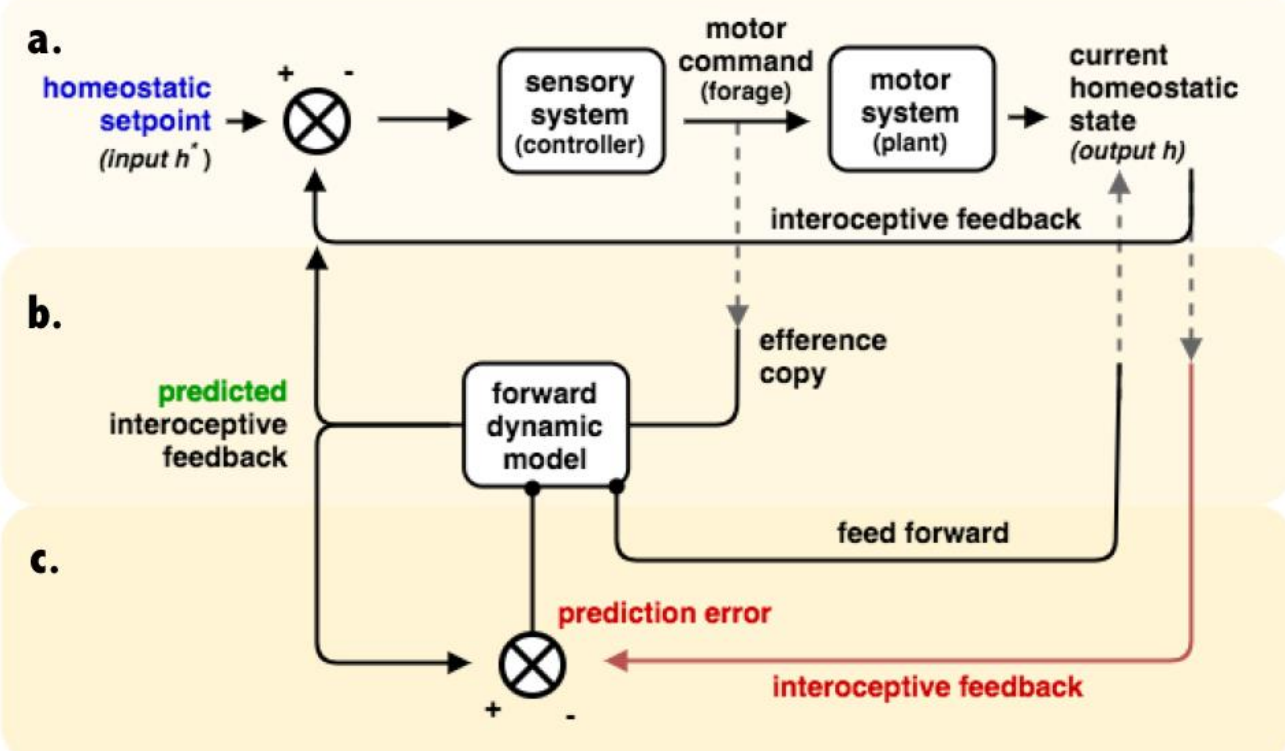

687 Figure 2 | Comparator-based models. Under this class of model, the agent is described as a homeostatic

688 error-correcting system. Broadly, the brain receives viscerosensory input from the body given its current

689 physiological state, and it computes the homeostatic error between its current state and its set point $\left(\mathrm{h}^{*}\right)$, and

690 then iteratively deploys controlled action to correct this. a, Depicts the subsystem that entails direct feedback

691 control, which combines a controller (here the sensory system), with a plant (here the motor system) that

692 executes action to influence the current homeostatic state. The current homeostatic state is sensed by

693 interoceptive feedback, which when compared to homeostatic set point, results in a homeostatic error that is

694 forwarded to the controller, from which further motor commands are sent to the plant to iteratively minimise

695 error. This is the homeostatic mechanism described by most physiology textbooks. b, An efference copy of the

696 motor command is sent to a forward dynamic model that predicts the future interoceptive feedback, given the

697 motor command. Residual errors between the predicted state and the set point are then iteratively minimised

698 with further commands. c, Finally, a prediction error, computed as the error between the predicted and the

699 current state is used to update the forward dynamic model. Insofar the system minimises this prediction error,

700 the forward dynamic model makes accurate predictions of the homeostatic consequences of its actions. 


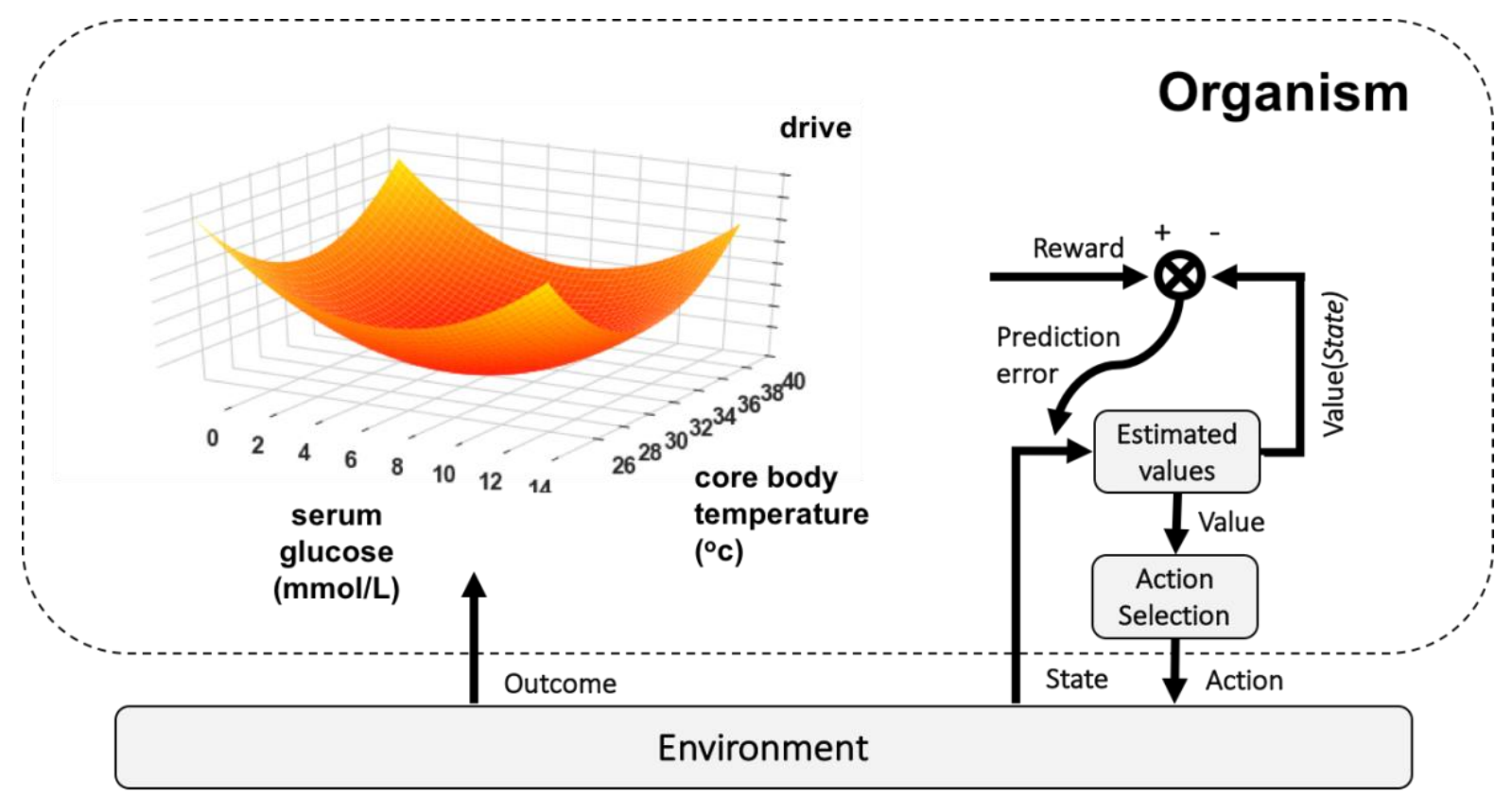

702 Figure $\mathbf{3}$ | Homeostatic reinforcement learning. In the upper left, the surface represents a drive function,

703 mapping from homeostatic state space (horizontal plane) to drive (vertical axis). The drive function depends

704 on the homeostatic state space, and the system to be modelled. Here, we illustrate a drive function based on

705 the surprise (negative log probability) derived from the survival probability function illustrated in Fig. 1b. If the

706 drive function is appropriately configured, then actions - that influence homeostatic state such that

707 homeostatic error is reduced - result in drive reduction. Under HRL, drive reduction is defined as rewarding, as

708 in drive reduction theory. By comparing the estimated value to the actual reward experienced (with negative

709 reward as drive inflation), a reward prediction error is generated and used to update future estimates of value.

710 Actions are selected as a function of these estimated values, such that selecting the actions that maximise

711 value, result in environmental exchanges that minimise drive, maximise reward, and thus minimise

712 homeostatic error. Adapted from (Keramati \& Gutkin 2014) with permission. 

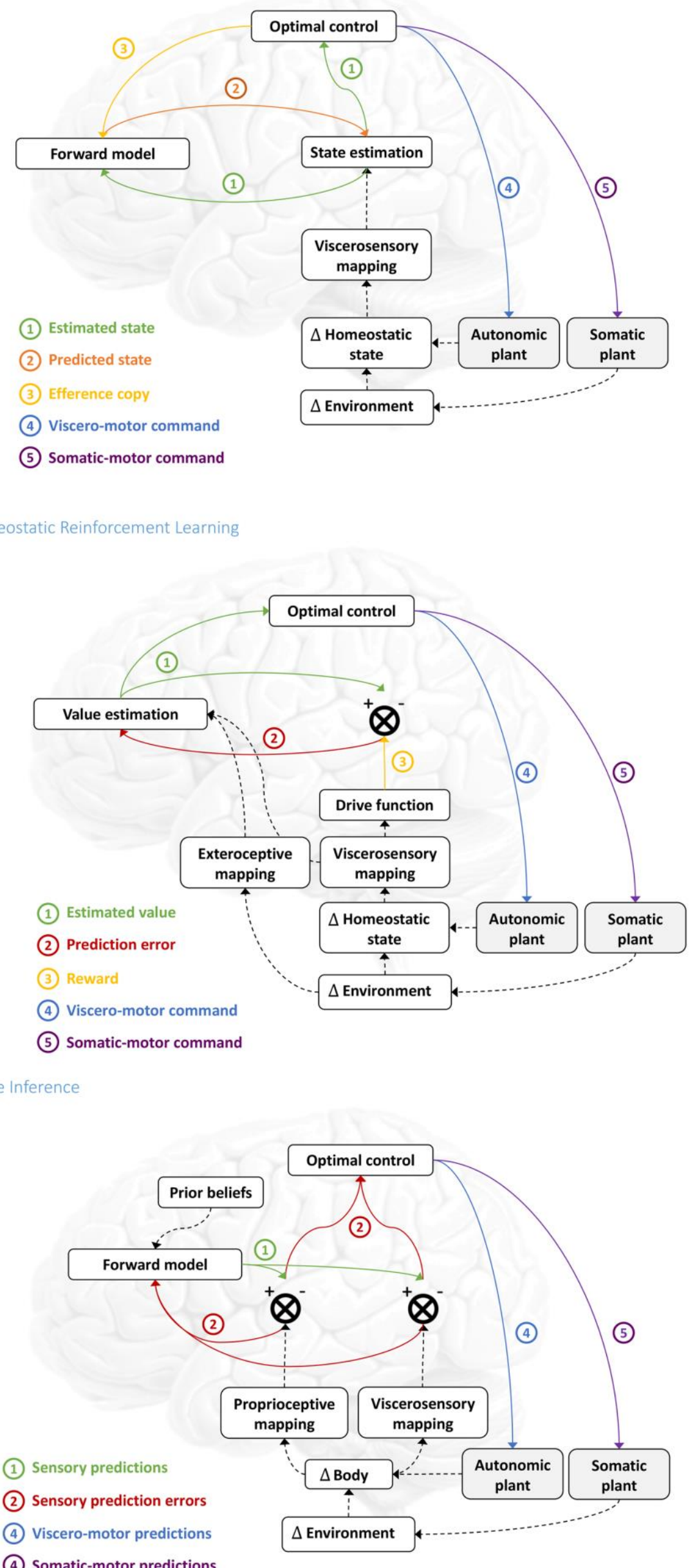
715 Figure 4 | Comparing models. Common to all model architectures is the fact that the agent is in a given

716 homeostatic state and a given environmental state. The agent can act in two ways. First it can engage in

717 physiological homeostasis by controlling its autonomic plant, which directly modulates the homeostatic state

718 of the body ( $\Delta$ homeostatic state). Second it can engage in behavioural homeostasis by moving, via the

719 somatic-motor system, to change its sampling of the environment ( $\Delta$ environment), which will indirectly

720 change its homeostatic state. These changes in homeostatic state are hidden, but they can be transformed

721 into neural input by the viscerosensory system (viscerosensory mapping). How the different theories prescribe

722 the control of these two plants based on sensory inputs is highlighted in the following comparison. Optimal

723 control theory. This is a schematic summary of the components commonly found in conventional treatments

724 of optimal motor control, here applied to homeostatic control. The hidden homeostatic states produce

725 interoceptive sensations through the viscerosensory mapping. This viscerosensory input is used for hidden-

726 state estimation (e.g. by Bayesian filtering) based on the forward model and a (weighted) prediction error. The

727 prediction error is the difference between sensory input and predictions of that input given the predicted state

728 (orange). The state estimates are used for optimal control, which returns motor commands (purple and blue)

729 that minimize future cost or loss, specified by a cost function (not shown, alternatively known as an inverse

730 model). These optimal control signals are then sent to the two motor plants and (through an efference copy,

731 yellow) to the forward model. The forward model then computes the predicted change in hidden homeostatic

732 states. In this scheme, the forward model can be regarded as a mapping from motor control to changes in

733 hidden homeostatic states. Effectively, its role is to finesse the problem of inferring homeostatic states and

734 thereby optimize homeostatic control signals. This is necessary because delays and noise on sensory signals

735 could easily confound the implicit closed-loop control used by this scheme. Homeostatic reinforcement

736 learning. Here we interpret the schematic in Fig. 3 using the same logic and terms wherever possible. Again,

737 we start with the hidden homeostatic states that are sensed via a viscerosensory mapping. This viscerosensory

738 input is submitted to a drive function, mapping from sensory state to the negative valenced motivational drive.

739 The drive reductions are encoded as experienced rewards (maroon), which are subject to a temporal

740 difference learning, where the value of sensory states are estimated as the rational expectations of future

741 discounted rewards following from that state. The difference between the value estimated at a given trial

742 (green), and the updates to that value based on the new sensory inputs (exteroceptive and viscerosensory),

743 yields a reward prediction error (red), that is used to update the value estimate. The value estimates (green)

744 for different possible actions, are then subject to action selection, from which the highest value action can be

745 probabilistically selected. Active inference. We start again by considering environmental dynamics caused by

746 somatic action. Along with autonomic action, this can result in changes to the body, causing both

747 proprioceptive and viscerosensory input (we omit exteroceptive sensations for clarity), yielding proprioceptive

748 and viscerosensory prediction errors. These prediction errors are simply the difference between the sensory

749 input observed and the sensory inputs predicted under the predicted (hidden) states. In this form, top-down

750 predictions from the forward model are compared with sensory inputs to produce bottom-up prediction errors

751 (red connections) that enter the forward model. Crucially, the mapping from hidden states to sensations is

752 now part of the forward (and thus, generative) model. Here, cost functions have been replaced by prior beliefs 
753 about (desired) homeostatic states. Allostatic regulation here can be achieved through prior beliefs over

754 homeostatic trajectories. These prior beliefs enter the forward model to guide predictions of sensory inputs.

755 These prior beliefs set the targets and priorities of homeostatic control, and thus are strongly selected as a

756 function of their contribution to survival (and thus fitness). Proprioceptive predictions are fulfilled by the

757 somatic motor system by classical motor reflex arcs (the somatic plant), while predictions of viscerosensory

758 input are fulfilled by the autonomic plant. Optimal control now reduces to simply suppressing proprioceptive

759 and viscerosensory prediction errors. 


\section{a.}

$$
p(h \mid s, m)=\frac{p(s \mid h, m) p(h \mid m)}{p(s \mid m)}
$$

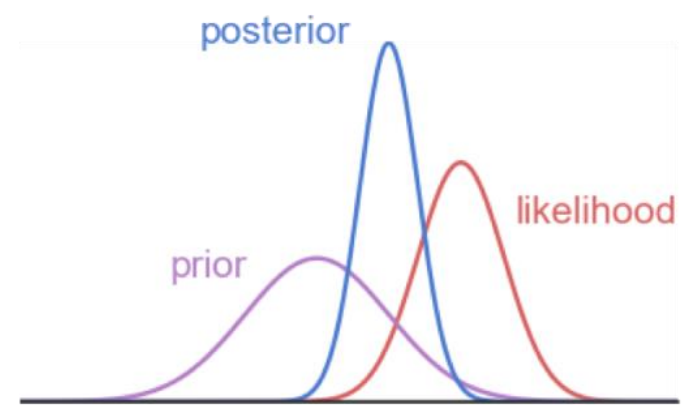

b.

Figure 5 | Interoception, Bayes rule and generative models. a, Bayes rule provides the probabilistic

foundation for the generative model $m$, which the agent embodies, and under which the agent expects homeostasis. This consists of a likelihood function $p(s \mid h, m)$ (a probabilistic map from hidden external states, $h$, to sensory inputs $s$ ) in conjunction with a prior $(h \mid m)$ (a probability distribution over external states, including bodily states). In this setting, the prior can be interpreted as a probabilistic set point. Calculating the posterior $p(h \mid s, m)$ is model inversion and yields the probability of a hidden homeostatic state, given the sensory input. Thus, the posterior is a mix between likelihood (how likely is this) and prior (how often does it occur) weighted by their relative precision (see Bogacz 2015). The denominator $p(s \mid m)$ is a normalisation term that ensures the posterior integrates to one. Importantly, this term is also the foundation of Bayesian model selection (see Ghahramani 2012 for an introduction). b, Illustrates how homeostatic dynamics (left box) that are hidden from the agent give rise to sensory input $s$, which the phenotype must infer on, given its generative model $m$. The causal structure of the external world (including the body) is encoded in synaptic activity (right box) encoded in a forward dynamic model, which allows inference about the causes (hidden states) of the sensory input. 
a.

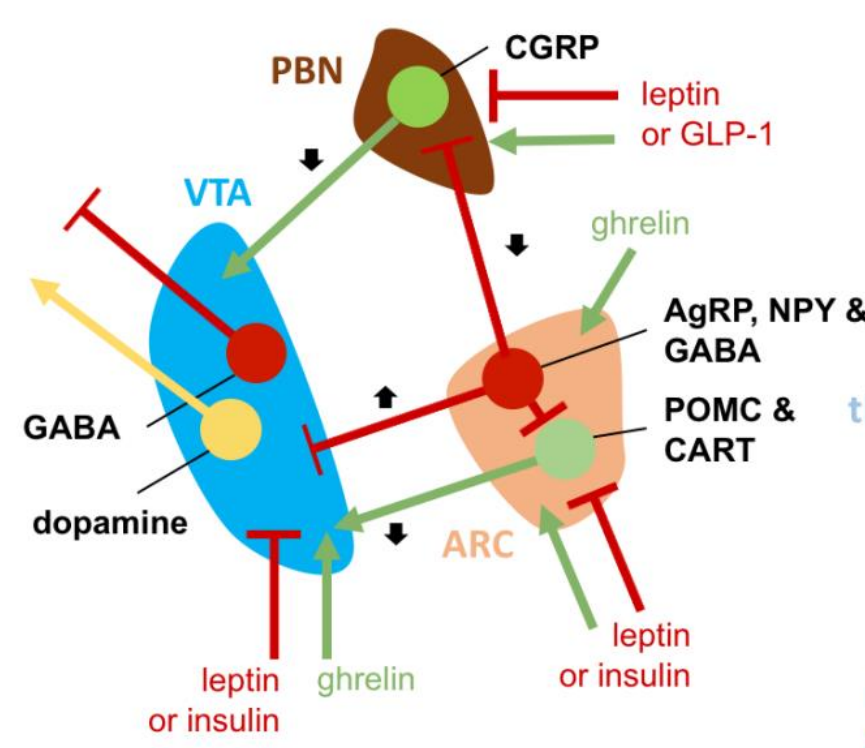

orexigenic

anorexigenic b.

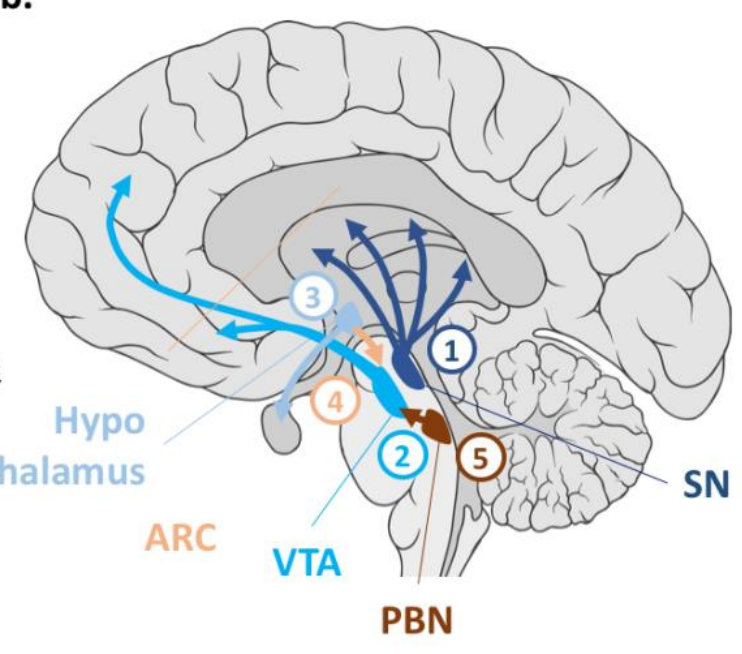

(1) Nigrostraital (Substantia Nigra Dorsal Straitum)

(2) Mesolimbic \& Mesocortical (Ventral Tegmental Area $\Rightarrow \begin{aligned} & \text { Ventral Straitum } \\ & \text { Prefrontal Cortex }\end{aligned}$

(3) Tuberoinfundibular (Hypothalamus $\Rightarrow$ Pituitary gland)

(4) Arcuate nucleus $\Rightarrow$ Ventral Tegmental Area

(5) Parabrachial nucleus $\Rightarrow$ Ventral Tegmental Area

Figure 6 | Homeostatic-reinforcement interface. a, Red T-lines and lettering illustrate inhibitory inputs; Green arrows and text indicate excitatory inputs. Dopamine is coloured yellow as this can have both excitatory and inhibitory effects depending on receptor subtypes. Projections: Cocaine and amphetamine regulated transcript (CART) and pro-opiomelanocortin (POMC) in the ARC of the hypothalamus process POMC to alpha-MSH that activate melanocortin-4 receptors (MC4R) on post-synaptic cells in the arcuate of the lateral hypothalamus (ARC), which projects to the VTA (details not shown here, see Ferrario et al. 2016). This melanocortin pathway is suppressed by neighbouring cells in the ARC that produce Agouti-related protein (AgRP), neuropeptide $Y$ (NPY) and GABA that all inhibit POMC neurons (Mandelblat-Cerf et al. 2015) and project to many of the same sites, including the VTA. Further, these project to CGRP neurons in the parabrachial nuclei, which in turn projects to VTA. Hormone input: AgRP neurons are inhibited (red T-bar) by leptin and insulin, whereas POMC are activated by those same hormones (green arrow). Hormone ghrelin, that signal short term energy deficits, activates AgRP and dopamine (green arrows) in the VTA (Palmiter 2007). Conversely, leptin and insulin attenuates dopaminergic firing (red T-bar). b.. There are four important dopaminergic pathways that project from the midbrain widely through the brain. Importantly, the VTA projects through the mesolimbic \& 
795

796

797

798

799

800

801

802

803

804

805

806

807

808

809

810

811

812

813

814

815

816

817

818

819

820

821

\section{Acknowledgements}

We thank Mehdi Keramati and Boris Gutkin for several helpful discussions. This work was supported by the following funders: H.R.S (Lundbeck Foundation Grant of Excellence "ContAct" ref: R59 A5399 ; Novo Nordisk Foundation Interdisciplinary Synergy Programme Grant “BASICS” ref: NNF14OC0011413) O.J.H (Lundbeck Foundation, ref: R140-2013-13057;

Danish Research Council ref: 12-126925) T.M. (Lundbeck Foundation ref: R140-2013-13057), K.F (The Wellcome Trust ref: 088130/Z/09/Z), D.B (The Francis Crick Institute, which receives its core funding from Cancer Research UK, the UK Medical Research Council, and the Wellcome Trust).

\section{Author contributions}

O.J.H and T.M conceived of the paper and made the figures. All authors contributed to the writing and editing of the paper.

\section{Author Information}

The authors declare no competing financial interests.

\section{References}

Aponte, Y., Atasoy, D. \& Sternson, S.M., 2011. AGRP neurons are sufficient to orchestrate feeding behavior rapidly and without training. Nature Neuroscience, 14(3), pp.351-355.

Atasoy, D. et al., 2012. Deconstruction of a neural circuit for hunger. Nature, 488(7410), pp.172-177.

Ballard, K. \& Knutson, B., 2009. Dissociable neural representations of future reward magnitude and delay during temporal discounting. Neurolmage, 45(1), pp.143-150.

Berridge, K.C., 2004. Motivation concepts in behavioral neuroscience. Physiology \& Behavior, 81(2), pp.179-209.

Berridge, K.C. \& Robinson, T.E., 2003. Parsing reward. Trends in Neurosciences, 26(9), pp.507-513.

Betley, J.N. et al., 2015. Neurons for hunger and thirst transmit a negative-valence teaching signal. Nature, 521(7551), pp.180-185. 
822

823

824

825

826

827

828

829

830

831

832

833

834

835

836

837

838

839

840

841

842

843

844

845

846

847

848

849

850

851

852

853

854

855

856

Bogacz, R., 2015. A tutorial on the free-energy framework for modelling perception and learning. Journal of Mathematical Psychology.

Carlson, J.N. et al., 1987. Selective enhancement of dopamine utilization in the rat prefrontal cortex by food deprivation. Brain Research, 400(1), pp.200-203.

Carpenter, R.H.S., 2004. Homeostasis: a plea for a unified approach. AJP: Advances in Physiology Education, 28(1-4), pp.180-187.

Chen, Y. \& Knight, Z.A., 2015. Making sense of the sensory regulation of hunger neurons. BioEssays : news and reviews in molecular, cellular and developmental biology, 38(4), pp.316-324.

Chen, Y. et al., 2016. Hunger neurons drive feeding through a sustained, positive reinforcement signal. elife, 5, p.e18640.

Cone, J.J. et al., 2016. Physiological state gates acquisition and expression of mesolimbic reward prediction signals. Proceedings of the National Academy of Sciences of the United States of America, 113(7), pp.1943-1948.

Dietrich, M.O. et al., 2012. AgRP neurons regulate development of dopamine neuronal plasticity and nonfood-associated behaviors. Nature Neuroscience, 15(8), pp.11081110.

Domingos, A.I. et al., 2011. Leptin regulates the reward value of nutrient. Nature Neuroscience, 14(12), pp.1562-1568.

Dranias, M.R., Grossberg, S. \& Bullock, D., 2008. Dopaminergic and non-dopaminergic value systems in conditioning and outcome-specific revaluation. Brain Research, 1238, pp.239-287.

Feldman, A.G., 1986. Once more on the equilibrium-point hypothesis (lambda model) for motor control. Journal of motor behavior, 18(1), pp.17-54.

Ferrario, C.R. et al., 2016. Homeostasis Meets Motivation in the Battle to Control Food Intake. Journal of Neuroscience, 36(45), pp.11469-11481.

Friston, K., 2012. A Free Energy Principle for Biological Systems. Entropy, 14(11), pp.21002121.

Friston, K. \& Ao, P., 2012. Free Energy, Value, and Attractors. Computational and Mathematical Methods in Medicine, 2012(5), pp.1-27.

Friston, K. et al., 2017. Deep temporal models and active inference. Neuroscience \& Biobehavioral Reviews.

Friston, K., Kilner, J. \& Harrison, L., 2006. A free energy principle for the brain. Journal of Physiology-Paris, 100(1-3), pp.70-87.

Frith, C., 2012. Explaining delusions of control: The comparator model 20years on. 
858

859

860

861

862

863

864

865

866

867

868

869

870

871

872

873

874

875

876

877

878

879

880

881

882

883

884

885

886

887

888

889

890

891

Fulton, S., 2000. Modulation of Brain Reward Circuitry by Leptin. 287(5450), pp.125-128. Available at: http://www.sciencemag.org/cgi/doi/10.1126/science.287.5450.125.

Ghahramani, Z., 2012. Bayesian non-parametrics and the probabilistic approach to modelling. Philosophical Transactions of the Royal Society A: Mathematical, Physical and Engineering Sciences, 371(1984), pp.20110553-20110553.

Glimcher, P.W., 2010. Foundations of Neuroeconomic Analysis, Oxford University Press.

Heffner, T.G., Hartman, J.A. \& Seiden, L.S., 1980. Feeding increases dopamine metabolism in the rat brain. Science (New York, N.Y.), 208(4448), pp.1168-1170.

Hull, C.L., 1943. Principles of Behavior: An Introduction to Behavior Theory, D. AppletonCentury Company, Incorporated.

Keramati, M. \& Gutkin, B., 2014. Homeostatic reinforcement learning for integrating reward collection and physiological stability. elife, 3, p.475.

King, C.M. \& Hentges, S.T., 2011. Relative number and distribution of murine hypothalamic proopiomelanocortin neurons innervating distinct target sites. PLOS ONE, 6(10), p.e25864.

Krashes, M.J. et al., 2014. An excitatory paraventricular nucleus to AgRP neuron circuit that drives hunger. Available at: http://www.nature.com/nature/journal/v507/n7491/abs/nature12956.html.

Luquet, S. et al., 2005. NPY/AgRP neurons are essential for feeding in adult mice but can be ablated in neonates. Science (New York, N.Y.), 310(5748), pp.683-685.

Mandelblat-Cerf, Y. et al., 2015. Arcuate hypothalamic AgRP and putative POMC neurons show opposite changes in spiking across multiple timescales. eLife, 4, p.351. Available at: http://elifesciences.org/lookup/doi/10.7554/eLife.07122.

McCullough, L.D. \& Salamone, J.D., 1992. Involvement of nucleus accumbens dopamine in the motor activity induced by periodic food presentation: a microdialysis and behavioral study. Brain Research, 592(1-2), pp.29-36.

Miall, R.C. \& Wolpert, D.M., 1996. Forward Models for Physiological Motor Control. 9(8), pp.1265-1279. Available at: http://www.sciencedirect.com/science/article/pii/S0893608096000354.

Niv, Y., Duff, M.O. \& Dayan, P., 2005. Behavioral and Brain Functions. Behavioral and Brain Functions, 1(1), pp.6-9.

Palmiter, R.D., 2007. Is dopamine a physiologically relevant mediator of feeding behavior? Trends in Neurosciences, 30(8), pp.375-381. Available at: http://linkinghub.elsevier.com/retrieve/pii/S0166223607001336. 
892

893

894

895

896

897

898

899

900

901

902

903

904

905

906

907

908

909

910

911

912

913

914

915

916

917

918

919

920

921

922

923

924

925

Pandit, R. et al., 2015. Central Melanocortins Regulate the Motivation for Sucrose Reward J. E. McCutcheon, ed. PLOS ONE, 10(3).

Pezzulo, G., Rigoli, F. \& Friston, K., 2015. Active Inference, homeostatic regulation and adaptive behavioural control. Progress in Neurobiology, 134, pp.1-19.

Powers, W.T., 2016. Perceptual Control Theory, Living Control Systems Publ.

Radhakishun, F.S., van Ree, J.M. \& Westerink, B.H., 1988. Scheduled eating increases dopamine release in the nucleus accumbens of food-deprived rats as assessed with online brain dialysis. Neuroscience letters, 85(3), pp.351-356.

Schultz, W., Dayan, P. \& Montague, P.R., 1997. A neural substrate of prediction and reward. Science (New York, N.Y.), 275(5306), pp.1593-1599.

Seth, A.K., 2013. Interoceptive inference, emotion, andthe embodied self. Trends in Cognitive Sciences, 17(11), pp.1-9.

Small, D.M., Jones-Gotman, M. \& Dagher, A., 2003. Feeding-induced dopamine release in dorsal striatum correlates with meal pleasantness ratings in healthy human volunteers. Neurolmage, 19(4), pp.1709-1715.

Stauffer, W.R., Lak, A. \& Schultz, W., 2014. Dopamine Reward Prediction Error Responses Reflect Marginal Utility. Current Biology, 24(21), pp.2491-2500.

Stephan, K.E. et al., 2016. Allostatic Self-efficacy: A Metacognitive Theory of Dyshomeostasis-Induced Fatigue and Depression. Frontiers in human neuroscience, 10, p.49.

Sterling, P., 2012. Allostasis: A model of predictive regulation. Physiology \& Behavior, 106(1), pp.5-15.

Takahashi, K.A. \& Cone, R.D., 2005. Fasting induces a large, leptin-dependent increase in the intrinsic action potential frequency of orexigenic arcuate nucleus neuropeptide Y/Agouti-related protein neurons. Endocrinology, 146(3), pp.1043-1047.

Thorndike, E.L., 1927. The law of effect. American Journal of Psychology, 39, pp.212-222.

Tobler, P.N., Fiorillo, C.D. \& Schultz, W., 2005. Adaptive coding of reward value by dopamine neurons. Science (New York, N.Y.), 307(5715), pp.1642-1645.

Woods, S.C. \& Ramsay, D.S., 2007. Homeostasis: beyond Curt Richter,

Yen, H.-H. \& Roseberry, A.G., 2015. Decreased consumption of rewarding sucrose solutions after injection of melanocortins into the ventral tegmental area of rats.

Psychopharmacology, 232(1), pp.285-294. 
926 Fiorillo, C. D., P. N. Tobler and W. Schultz (2003). "Discrete coding of reward probability

927 and uncertainty by dopamine neurons." Science 299(5614): 1898-1902.

928 Friston, K., P. Schwartenbeck, T. FitzGerald, M. Moutoussis, T. Behrens and R. J. Dolan

929 (2014). "The anatomy of choice: dopamine and decision-making." Philos Trans R Soc Lond

930 B Biol Sci 369(1655).

931 Humphries, M. D., M. Khamassi and K. Gurney (2012). "Dopaminergic Control of the

932 Exploration-Exploitation Trade-Off via the Basal Ganglia." Front Neurosci 6: 9.

933 Niv, Y., M. O. Duff and P. Dayan (2005). "Dopamine, uncertainty and TD learning." Behav

934 Brain Funct. 1: 6.

935 Schwartenbeck, P., T. H. FitzGerald, C. Mathys, R. Dolan and K. Friston (2015). "The

936 Dopaminergic Midbrain Encodes the Expected Certainty about Desired Outcomes." Cereb

937 Cortex 25(10): 3434-3445.

938 Seth, A. (2014). The cybernetic brain: from interoceptive inference to sensorimotor

939 contingencies. MINDS project. Metzinger, T; Windt, JM, MINDS.

940 